\title{
Isolation and Purification of an Antibiotic Polyketide JBIR-99 from the Marine Fungus Meyerozyma guilliermondii by High-Speed Counter-Current Chromatography
}

\author{
Hankui Wu ${ }^{1,}$, , Zhen $\mathrm{Su}^{1}$, Jianguang Chen ${ }^{1}$, Yuanping Wang ${ }^{1}$, Xinyong You ${ }^{1}$, Kunpeng Zhang ${ }^{1, *}$, \\ Xiaobin $\mathbf{L i}^{2, *}$
}

${ }^{1}$ School of Biotechnology and Food Engineering, Anyang Institute of Technology, Anyang, China

${ }^{2}$ Biology Institute, Qilu University of Technology (Shandong Academy of Sciences), Jinan, China

Email address:

wuhankui222@126.com (Hankui Wu),1095557379@qq.com (Kunpeng Zhang),bin85666666@163.com (Xiaobin Li)

${ }^{*}$ Corresponding author

\section{To cite this article:}

Hankui Wu, Zhen Su, Jianguang Chen, Yuanping Wang, Xinyong You, Kunpeng Zhang, Xiaobin Li. 1solation and Purification of an Antibiotic Polyketide JBIR-99 from the Marine Fungus Meyerozyma guilliermondii by High-Speed Counter-Current Chromatography. International Journal of Pharmacy and Chemistry. Vol. 7, No. 4, 2021, pp. 52-57. doi: 10.11648/j.ijpc.20210704.11

Received: June 28, 2021; Accepted: July 7, 2021; Published: July 15, 2021

\begin{abstract}
JBIR-99 is a natural compound isolated from marine source which possesses strong antibiotic activity. An efficient approach using a combination of size exclusion chromatography with a Sephadex LH-20 and high-speed counter-current chromatography (HSCCC) has been successfully developed for the isolation and purification of a polyketide from the solid-state fermentation of Meyerozyma guilliermondii, a strain of marine fungus isolated from Indian Ocean. The active compound was isolated with purity $>95 \%$ by HSCCC using an optimized solvent system composed of petroleum ether-ethyl acetate- $95 \%$ ethanol-water (5:3:5:3, v/v/v/v) after size exclusion chromatography. This compound was successfully purified in the quantity of $68 \mathrm{mg}$ from $120 \mathrm{mg}$ of the crude extract. The structure of JBIR-99 was elucidated and assigned by 1D, 2D NMR spectroscopic, and positive HRESITOFMS. Moreover, the relative configuration of compound JBIR-99, displaying a quite complex multi-ring structure, is determined by X-ray crystallography for the first time. The purification method developed for JBIR-99 will facilitate the further investigation and development of this antibiotic agent as a lead compound. Furthermore, it is suggested that the combination of size exclusion chromatography and HSCCC could be more widely applied for the isolation and purification of polyketides from marine fungi.
\end{abstract}

Keywords: JBIR-99, HSCCC, Antibiotics, X-ray Crystal

\section{Introduction}

Polyketides are a class of secondary metabolites produced by certain living organisms. Many polyketides, such as geldanamycin, doxycycline, erythromycin A, clarithromycin, and azithromycin et al have antimicrobial properties and tacrolimus has immunosuppressive property, and many mycotoxins produced by fungi are polyketides too. Structurally, polyketides are complex organic compounds that are often highly active biologically [1]. Meyerozyma guilliermondii (formerly known as Pichia guilliermondii until its rename in 2010) is a species of yeast of the genus Meyerozyma whose asexual or anamorphic form is known as Candida guilliermondii. C. guilliermondii has been isolated from numerous human infections [2]. JBIR-99 (1; Figure 1) is a recently reported xanthoquinodin-like compound isolated from marine sponge-derived fungus Tritirachium $s p$. SpB081112MEf2 [3], then $\mathrm{Wu}$ et al [4] reported the isolation of this compound from the marine fungus Engyodontium album strain LF069. The compound exhibited inhibitory activity against methicillin resistant Staphylococcus aureus (MRSA) that was 10-fold stronger than chloramphenicol. It is a promising compound for further investigation. However, the reported isolation and purification of JBIR-99 has included multiple column chromatography steps and preparative HPLC, which is time-consuming, leads to the loss of target compound due to degradation during the long process, and is not industrially viable because of the cost of 
solid supports (silica gel or resin) for separation. Accordingly; these techniques are not typically suitable for purification of large quantities of material unless no other methods can complete the task [5-6]. Alternatively, high-speed counter-current chromatography (HSCCC) is a liquid-liquid separation chromatography that can reduce the separation time and cost, and provide effectively total sample recovery due to the lack of a solid support matrix that can degrade or permanently retain target molecules [7]. HSCCC has recently been applied to the isolation of various natural products, most typically coming from marine natural products [8-9]. The purpose of this study was to develop an efficient method for the preparative isolation and purification of JBIR-99 using the combined methods Sephadex LH-20 and HSCCC, which are both nondestructive and nonabsorptive techniques.

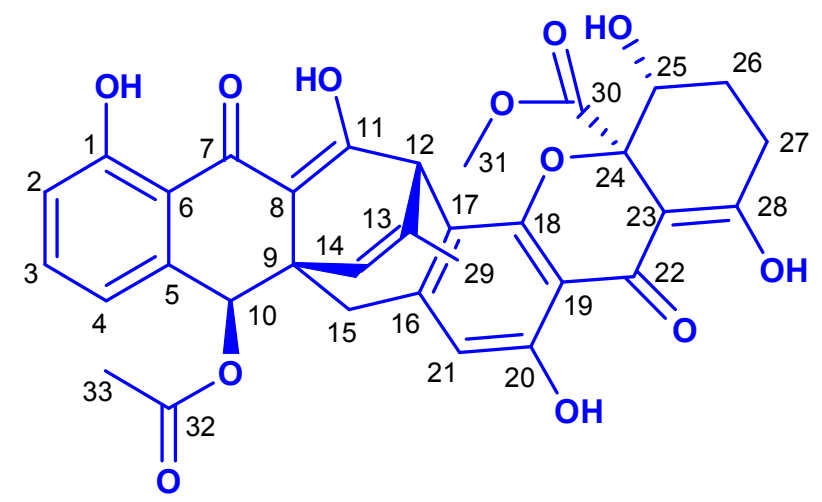

Figure 1. Structure of JBIR-99.
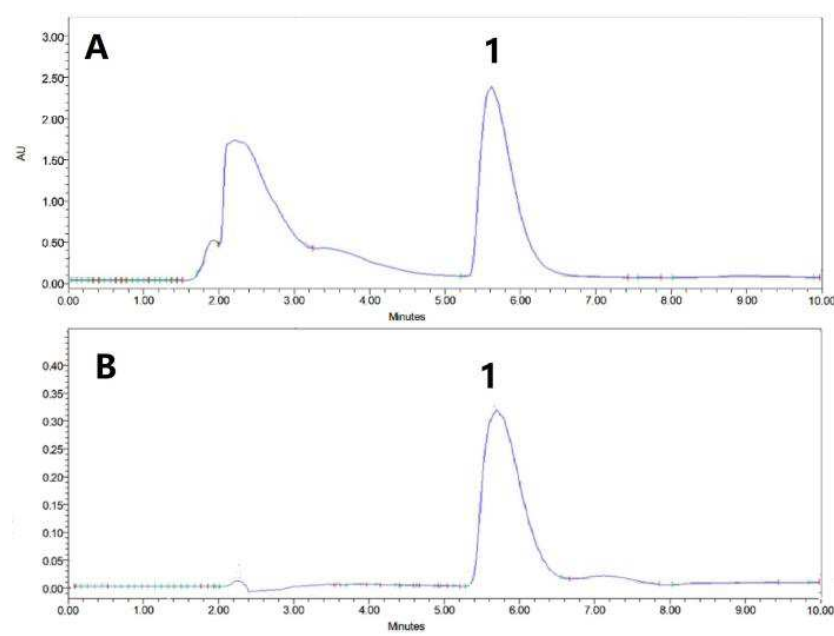

Figure 2. Representative high-performance liquid chromatography (HPLC) chromatograms $(220 \mathrm{~nm})$ of samples prepared from M. guilliermondii (A). Fractions 67-71 from the size exclusion chromatography of the crude acetonitrile layer; (B) Subfraction that contains 1 after preparative separation by high-speed counter-current chromatography (HSCCC).

\section{Experimental}

\subsection{Reagents and Materials}

All solvents used for HSCCC were of analytical grade (Fuyu Chemical Co., Ltd., Tianjin, China). $95 \%$ ethanol was bought from Hebei Ruikang Medical Science and Technology Co., Ltd.
(Hengshui, China). Reverse osmosis Milli-Q water (18 M) (Millipore, Bedford, MA, USA) was used for all solutions and dilutions. Acetonitrile used for HPLC analyses was of chromatographic grade and purchased from Shanghai Aladdin Bio-Chem Technology Co., Ltd (Shanghai, China). The $\mathrm{CD}_{3} \mathrm{COCD}_{3}$ used for NMR analyses was purchased from Tenglong Weibo Technology (Qingdao, China). The fungus Y39-1 was was kindly provided by First Institute of Oceanography State Oceanic Administration (SOA) Qingdao, China, which was isolated from sea water samples collected from the Indian Ocean at a depth of about $30 \mathrm{~m}\left(88^{\circ} 59^{\prime} 51^{\prime \prime} \mathrm{E}, 2^{\circ} 59^{\prime} 54^{\prime \prime} \mathrm{S}\right)$.

\subsection{Apparatus}

HSCCC was carried out using an OptiChrome-500 PLUS high-speed counter-current chromatograph (Counter Current Technology Co., Ltd., Jiangyin, China) equipped with three multilayer coil separation columns. The three multilayer coil columns were connected in series and had a combined volume of $500 \mathrm{~mL}$. The inner diameter (i. d.) of the tubing was $2.1 \mathrm{~mm}$, and the sample loop contained $30 \mathrm{~mL}$. The revolution radius was $5 \mathrm{~cm}$, and the $\beta$ values of the multilayer coils varied from 0.6 at the internal terminal to 0.8 at the external terminal. The rotational speed of the apparatus was regulated with a speed controller in the range of 0 to $1200 \mathrm{rpm}$. The apparatus was also equipped with one P-3000 metering pumps (Beijing Tong Heng Innovation Technology Co., Ltd, Beijing, China), an HD-3 UV detector (Heqin Analytical Instrument Co., Ltd Shanghai, China.), and an N2000 data analysis system (Institute of Automation Engineering, Zhejiang University, Hangzhou, China) was employed for HSCCC data collection and analysis. The HPLC equipment was using a Waters Xbridge ${ }^{\mathrm{TM}} \mathrm{C} 18$ column $(250 \mathrm{~mm} \times 4.6 \mathrm{~mm}, 5 \mu \mathrm{m}$ particle size $)$ equipped with a UV 3000 spectrometer (Beijing Tong Heng Innovation Technology Co., Ltd, Beijing, China), a CXTH-3000 workstation (Beijing Tong Heng Innovation Technology Co., Ltd, Beijing, China). NMR experiments including ${ }^{1} \mathrm{H}, 13 \mathrm{C}$, DEPT, ${ }^{1} \mathrm{H}-{ }^{1} \mathrm{H}$ COSY, HMQC, and HMBC were carried out using a $400 \mathrm{MHz}$ Bruker Avance nuclear magnetic resonance spectrometer (Bruker Biospin, Rheinstetten, Germany) spectrometer. HR-ESI-MS data was measured using a Waters HRESITOF Premier LC/MS spectrometer (Waters Co., Milford, MA, USA). Column chromatography (CC) was carried out with Sephadex LH-20 (Pharmacia, Uppsala, Sweden).

\subsection{Fungal Materials}

The fungus Y39-1 was stored at $4^{\circ} \mathrm{C}$ on PDA slants as provided by one of the co-authors (Dr. X. L.). Fungal identification was carried out by Shandong Lige Technology Co., Ltd. (Jinan, China) using a molecular biological protocol by DNA amplification and sequencing of the ITS region The sequence data of the fungal strain was most similar (100\%) to the sequence of Meyerozyma guilliermondii. A voucher strain was deposited at the Biology Institute, Qilu University of Technology (Shandong Academy of Sciences) with the access code Y39-1. 


\subsection{Preparation of Crude Sample from Y39-1 for HSCCC}

The fungus Y39-1 on PDA slants was cut into small pieces and grown under static conditions at $25^{\circ} \mathrm{C}$ for 35 days in a solid autoclaved rice substrate medium containing $80 \mathrm{~g}$ of rice and $120 \mathrm{~mL}$ of water in $500 \mathrm{~mL}$ flasks (30 flasks in all). After incubation, the mycelia and solid rice were extracted with EtOAc, and the extracts were concentrated to yield $12.8 \mathrm{~g}$ of residue under reduced pressure. The residue was partitioned between $200 \mathrm{~mL}$ of petroleum ether and $200 \mathrm{~mL}$ of acetonitrile the lower layer was concentrated to yield $2.2 \mathrm{~g}$ of residue, which was subjected to a glass column packed with Sephadex LH-20 and eluted with 95\% ethanol to obtain 100 fractions. Fractions 67-71 were combined based on TLC monitoring and used for later HSCCC separation.

\subsection{Preparation of Solvent System and Sample Solution}

The HSCCC experiments were performed using a two-phase solvent system comprising petroleum ether/ethyl acetate $/ 95 \%$ ethanol/water (5: 3: $5: 3, \mathrm{v} / \mathrm{v} / \mathrm{v} / \mathrm{v}$ ) solvent. The two phases were separated after thoroughly equilibrating the mixture in a separating funnel at room temperature. The upper organic phase was used as the stationary phase, and the lower aqueous phase was employed as the mobile phase.

\subsection{HSCCC Separation}

The organic stationary phase (upper phase) was pumped into the HSCCC column (flow $30 \mathrm{~mL} / \mathrm{min}$ ), after it was full, the mobile phase was pumped into the column $(8 \mathrm{~mL} / \mathrm{min})$ while in the normal rotating mode $(1100 \mathrm{rpm})$. When the mobile phase emerged from the column, it indicated that hydrodynamic equilibrium had been achieved. The sample (fractions 67-71, $120 \mathrm{mg}$ ) obtained from the Sephadex LH-20 column chromatography was dissolved in $10 \mathrm{~mL}$ of a $1: 1(\mathrm{v} / \mathrm{v})$ mixture of the two HSCCC solvent system phases and injected to the sample port. The effluent from the HSCCC was monitored by UV at $220 \mathrm{~nm}$, and peaks were collected.

\subsection{Analysis and Identification}

The fraction generated by preparative HSCCC was evaluated by HPLC (50\% acetonitrile, flow $1 \mathrm{~mL} / \mathrm{min}$, monitored at $220 \mathrm{~nm})$. Peak $4\left(t_{R}=31 \mathrm{~min}\right)$ showed only one peak in the HPLC chromatogram, which was concentrated under reduced pressure to yield compound 1 (68 $\mathrm{mg})$. The purity of compound 1 was $95.21 \%$.

\section{Results and Discussion}

\subsection{Enrichment of JBIR-99 by Sephadex LH-20}

In order to remove the large amounts of pigments and other unknown compounds contained in the crude acetonitrile extract of $M$. guilliermondii was first subjected to size exclusion chromatography on a Sephadex LH-20 gravity column. The LH-20 column was eluted with ethanol (95\%). A total of 100 fractions (25 mL each) were successively collected, Fractions 67-71 were combined and determined to contain 1 in high quantity, and this sample was concentrated to dryness and stored in a refrigerator for later HSCCC separation.

\subsection{Optimization of HPLC Analysis for JBIR-99}

A high-performance liquid chromatography (HPLC) method was developed to ensure the baseline separation of the target compound and impurities, and evaluate the size exclusion chromatography fractions. Different flow rates, elution modes, detection wavelengths were screened. The result indicated that the target compound was baseline separated with acetonitrile-water $(50 \%$, containing $0.1 \%$ formic acid) as the solvent system, when the flow rate, column temperature and detection wavelength were set at $1.0 \mathrm{~mL} / \mathrm{min}$, $25^{\circ} \mathrm{C}$ and $220 \mathrm{~nm}$. Preliminary assignment of 1 in the chromatogram was made by comparison of peak retention time with reported reference [4]. The HPLC chromatogram of fractions showed the major, but not only peak, as being 1 (Figure 2A).

\subsection{Selection of the Solvent System for HSCCC}

Satisfactory HSCCC purification relies on two immiscible liquids to serve as stationary and mobile phases, the selection of a suitable biphasic solvent system plays a vital role in successful separations. It has been suggested that the partition coefficient $(\mathrm{K})$ is the most important parameter in solvent system selection, which should be $0.5 \leq \mathrm{K} \leq 2$ (close to 1 , best) to get a good separation for HSCCC in a suitable run time [10]. As previously reported in the literature [11], the two-phase solvent system "HEMW", comprising n-hexane-ethyl acetate-methanol-water, has been widely applied in the separation of natural products by HSCCC. In our study, to green the process of isolation, petroleum ether and $95 \%$ ethanol were used to replace n-hexane and methanol respectively.

Six sets of different proportional two-phase petroleum ether/ethyl acetate/95\% ethanol/water (PEEW) solvent systems were carried out to determine the partition value, $\mathrm{K}$, of the target compound at various volume ratios of petroleum ether/ethyl acetate/95\% ethanol/water (4: $4: 4: 4,3: 5: 4: 4,2$ : 6: $2: 6,1: 7: 1: 7,5: 3: 5: 3,6: 2: 6: 2$, all v/v/v/v) by HPLC analysis of each partition. The results, shown in Table 1 , indicated that the two-phase solvent system of $5: 3: 5: 3$ PEEW, v/v/v/v, provided a suitable partition value for JBIR-99 of $\mathrm{K}=0.58$ with good resolution and short elution time.

Table 1. $K$ values of compound 1 in different ratios of the PEEW at solvent system.

\begin{tabular}{lll}
\hline Solvent System & Ratios (v/v/v/v) & $\boldsymbol{K}$ \\
\hline $\mathrm{PE} / \mathrm{EtOAc} / 95 \% \mathrm{EtOH} /$ water & $4: 4: 4: 4$ & 2.07 \\
$\mathrm{PE} / \mathrm{EtOAc} / 95 \% \mathrm{EtOH} /$ water & $3: 5: 4: 4$ & 23.99 \\
$\mathrm{PE} / \mathrm{EtOAc} / 95 \% \mathrm{EtOH} /$ water & $2: 6: 2: 6$ & 56.69 \\
$\mathrm{PE} / \mathrm{EtOAc} / 95 \% \mathrm{EtOH} /$ water & $1: 7: 1: 7$ & 26.63 \\
$\mathrm{PE} / \mathrm{EtOAc} / 95 \% \mathrm{EtOH} /$ water & $5: 3: 5: 3$ & 0.58 \\
$\mathrm{PE} / \mathrm{EtOAc} / 95 \% \mathrm{EtOH} /$ water & $6: 2: 6: 2$ & 0.25 \\
\hline
\end{tabular}




\subsection{HSCCC Separation}

The selected fractions 67-71 from the size exclusion chromatography of the extract from C. guilliermondii $(120 \mathrm{mg})$ was applied for HSCCC separation with the chosen two-phase solvent system, petroleum ether/ethyl acetate $/ 95 \%$ ethanol/water (5: 3: 5: 3). In order to optimize the resolution and reduce the separation time, different flow rates and rotation speeds were evaluated. It was found that when the flow rate was $8 \mathrm{~mL} / \mathrm{min}$ and rotation speed was $1100 \mathrm{rpm}$, a good separation was achieved for elution of 1 with a good stationary phase retention of $70 \%$. The HSCCC peak fraction corresponding to $1(68 \mathrm{mg})$ was collected and determined to have purity of $95.12 \%$ by HPLC analysis (Figure 2B). The resulting HSCC chromatogram is shown in Figure 3, demonstrating the good resolution and peak shape of compound 1 at $t_{R}=31 \min ($ peak 4$)$.

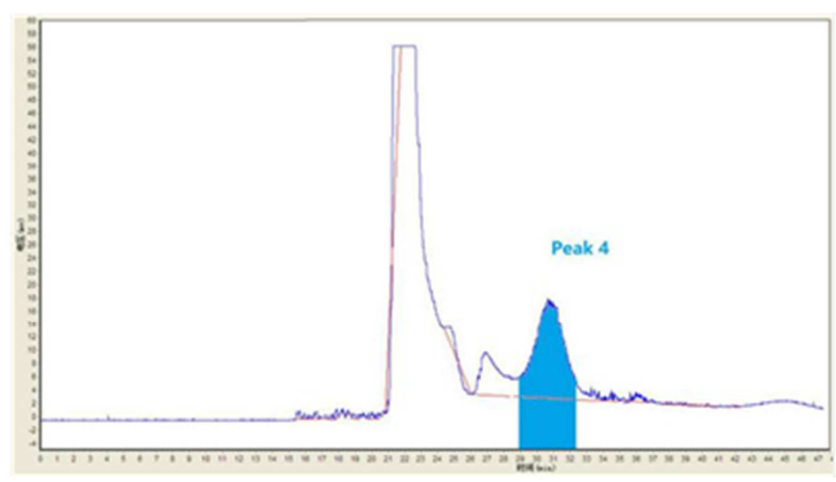

Figure 3. HSCCC chromatogram.

\subsection{Identification of Chemical Structure}

Compound 1 was identified by HR-ESI-MS, ${ }^{1} \mathrm{H}-\mathrm{NMR}$, ${ }^{13} \mathrm{C}-\mathrm{NMR}$, DEPT, ${ }^{1} \mathrm{H}-{ }^{1} \mathrm{H}$ COSY, HMQC and HMBC after purification by HSCCC, and its detailed data are shown in Table 2. The HMBC correlations are shown in Figure 2. Its molecular formula $\mathrm{C}_{33} \mathrm{H}_{28} \mathrm{O}_{12}$ was deduced by
HR-ESI-TOF-MS data at $m / z 639.1472[\mathrm{M}+\mathrm{Na}]+$. Compound 1 was identified as a xanthoquinodin-like compound, JBIR-99, with the chemical structure as shown in Figure 1. The structure of JBIR-99 was first disclosed by Ueda J. [3], but the single-crystal X-ray diffraction data were reported here for the first time.

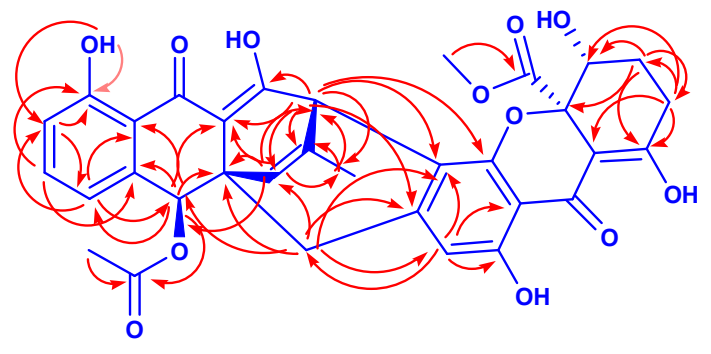

Figure 4. HMBC correlations of JBIR-99 (1).

\subsection{X-ray Crystallography}

X-ray crystal data for 1 . Colorless crystals of 1 were obtained in methanol. Crystal data (CCDC 1945666) were collected on a Bruker Smart APEX II diffractometer equipped with graphite-monochromitized Mo-K $\alpha$ radiation (Wavelength $=0.71073$ ). Orthorhombic, space group $\mathrm{P} 2{ }_{1} 2_{1} 2_{1}$ (no. 19), $\mathrm{a}=7.9628$ (7) $\AA, \mathrm{b}=15.1359$ (16) $\AA, \mathrm{c}=25.686$ (2) $\AA$, $\alpha=90, \beta=90, \gamma=90, V=3095.8$ (5)) $\AA^{3}, \mathrm{Z}=4, \mathrm{~T}=296 \mathrm{~K}, \mu$ $(\mathrm{Mo}-\mathrm{K} \alpha)=0.108 \mathrm{~mm}^{-1}$, Dcalc $=1.389 \mathrm{~g} / \mathrm{cm}^{3}, \mathrm{~F}(000)=1356$, $\mathrm{R} 1=0.0510, \mathrm{wR} 2=0.1466, \mathrm{GOF}=1.007$. Crystal dimensions $0.12 \times 0.10 \times 0.10 \mathrm{~mm}^{3}$. The total number of independent reflections measured was 5443, of which 3907 were observed. The structure was solved by direct method and refined by full-matrix least-squares on F2 using SHELX-2017 [12]. All non-hydrogen atomic positions were located in Fourier maps and refined anisotropically, while all of the hydrogen atoms were refined with isotropic displacement parameters (Figure 5). Moreover, these results are consistent with the relative configuration of 1 that was proposed in [3-4] on the basis of NMR data and X-ray crystal data.

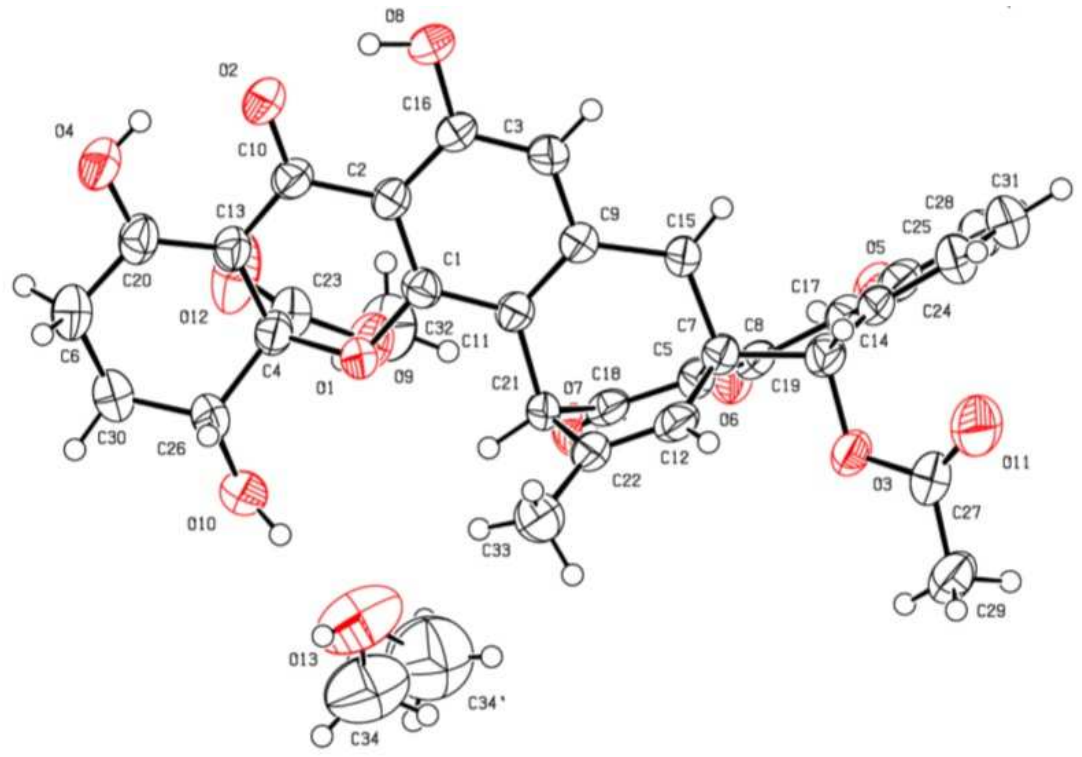

Figure 5. X-ray structure of JBIR-99 (1). 
Table 2. NMR data of JBIR-99 (1) in $\mathrm{CD}_{3} \mathrm{COCD}_{3}$

\begin{tabular}{|c|c|c|c|c|c|}
\hline Pos. & $\delta_{H}$ & $\boldsymbol{\delta}_{\mathrm{C}}$ & Pos. & $\boldsymbol{\delta}_{\mathrm{H}}$ & $\boldsymbol{\delta}_{\mathrm{C}}$ \\
\hline 1 & & 162.6 & 18 & & 156.8 \\
\hline 2 & $7.00, \mathrm{~d}(8.4)$ & 119.7 & 19 & & 105.6 \\
\hline 3 & $7.59, \mathrm{t}(8.4)$ & 137.1 & 20 & & 160.9 \\
\hline 4 & $7.13, \mathrm{~d}(8.4)$ & 122.9 & 21 & $6.17, \mathrm{~s}$ & 114.4 \\
\hline 5 & & 137.0 & 22 & & 188.2 \\
\hline 6 & & 116.3 & 23 & & 100.8 \\
\hline 7 & & 187.5 & 24 & & 86.5 \\
\hline 9 & & 42.1 & 26 & $2.27,2.09, \mathrm{~m}$ & 25.7 \\
\hline 10 & $6.05, \mathrm{~s}$ & 73.8 & 27 & $2.65, \mathrm{~m}$ & 28.5 \\
\hline 11 & & 187.8 & 28 & & 180.4 \\
\hline 12 & $4.95, \mathrm{~s}$ & 43.4 & 29 & $1.94, \mathrm{~s}$ & 20.6 \\
\hline 13 & & 142.9 & 30 & & 170.4 \\
\hline 14 & $5.78, \mathrm{~s}$ & 126.2 & 31 & $3.66, \mathrm{~s}$ & 53.1 \\
\hline 15 & $2.92, \mathrm{~d}(17.8), 2.72, \mathrm{~d}(17.8)$ & 36.2 & 32 & & 171.5 \\
\hline 16 & & 149.5 & 33 & $2.07, \mathrm{~s}$ & 20.9 \\
\hline
\end{tabular}

\section{Conclusions}

A rapid method relying on HSCCC after size exclusion chromatography on Sephadex LH-20 was utilized to separate JBIR-99 from the extract of the fungus $C$. guilliermondii in a lossless procedure. It was important to pre-treat the crude extract by partition between two phase solvents and pass through a Sephadex LH-20 column for HSCCC separation to improve the resolution and efficiency. The solvent system of petroleum ether/ethyl acetate $/ 95 \%$ ethanol/water (5: $3: 5: 3$, $\mathrm{v} / \mathrm{v} / \mathrm{v} / \mathrm{v}$ ) was optimized to isolate JBIR-99 (1). The separation condition was selected as follow: flow rate $8.0 \mathrm{~mL} / \mathrm{min}$, rotary speed $1100 \mathrm{rpm}$, column temperature $25^{\circ} \mathrm{C}$. Under the optimized HSCCC condition, $68 \mathrm{mg}$ of JBIR-99 with the high purity of $95.21 \%$ was isolated from $120 \mathrm{mg}$ of fractions $67-71$ of C. guilliermondii. This is the first report of the isolation of JBIR-99 by integrating size exclusion chromatography and HSCCC, and this method could be used for the effective isolation of different xanthoquinodin-like polyketides from different natural materials. This convenient and economical approach will be applicable for scale-up production of JBIR-99 to increase the yield. The purification procedure optimized for JBIR-99 will also facilitate the further development of this antibiotic agent as a lead compound and further structure-activity relationship studies.

Because we have checked that the fermentation extracts of the fungus $M$. guilliermondii had pro-angiogenetic activity by using zebrafish model. Our follow-up study will focus on the evaluation of the effects of the isolated compound JBIR-99 on the cardiovascular system and unveiling the possible mechanism of action.

\section{Acknowledgements}

This research was funded by NSFC (grant number 81602982) and the Doctoral Research Foudation of Anyang Institute of Technology (BSJ2021031), Key Technologies R \& D Program of Henan Province (212102311024).

\section{References}

[1] Huffman, J., Gerber, R., Du, L (2010). Recent advancements in the biosynthetic mechanisms for polyketide derived mycotoxin. Biopolymers, 93, 764-776.

[2] Wahengbam, R., Santosh, K., Giasuddin, A. and Kumaraswamy, J. (2014). Reliable differentiation of Meyerozyma guilliermondii from Meyerozyma caribbica by internal transcribed spacer restriction fingerprinting. BMC Microb. 14, 52-62.

[3] Ueda, J., Shinya, K. (2010). New xanthoquinodin-like compounds JBIR-97 -98 and -99 obtained from marine sponge-derived fungus Tritirachium $s p$, SpB081112MEf2. J. Antibioti., 2010, 63, 615.

[4] Wu, B., Wiese, J., Wenzel-Storjohann, A., Malien, S., Schmaljohann, R., Imhoff, J. F. (2016). Engyodontochones antibiotic polyketides from the marine fungus Engyodontium album Strain LF069. Chem. Eur. J., 2016, 22, 7452.

[5] Shibata, T.; Ishimaru, K.; Kawaguchi, S.; Yoshikawa, H.; Hama, Y. (2008). Antioxidant activities of phlorotannins isolated from Japanese Laminariacea. J. Appl. Phycol., 20, 705-711.

[6] Nakai, M., Kageyama, N., Nakahara, K., Miki, W. (2006). Phlorotannins as radical scavengers from the extract of Sargassum ringgoldianum. Mar Biotechnol. 8, 409-414.

[7] Duan, W., Ji, W., Wei, Y., Zhao, R., Chen, Z., Geng, Y., Jing, F., Wang, X. (2018). Separation and purification of fructo-oligosaccharide by high-speed counter-current chromatography coupled with precolumn derivatization. Molecules, 23, 381.

[8] Zhou, X., Yi, M., Ding, L., He, S., Yan, X. (2019). Isolation and purification of a neuroprotective phlorotannin from the marine algae Ecklonia maxima by Size Exclusion and high-speed counter-current chromatography. Mar drugs. 17, 212-219.

[9] Liu, Y., Zhou, X., Naman, C. B., Lu, Y., Ding, L., He, S. (2018). Preparative separation and purifification of trichothecene mycotoxins from the marine fungus Fusarium sp. LS68 by high-speed countercurrent chromatography in stepwise elution mode. Mar drugs. 16, 73. 
[10] Wang, J., Gu, D., Wang, M., Guo, X., Li, H., Dong, Y., Guo, H., Wang, Y., Fan, M., Yang, Y. (2017). Rational approach to solvent system selection for liquid-liquid extraction-assisted sample pretreatment in counter-current chromatography. J Chromatogr B. 1053, 16-19.

[11] Shaheen, N., Lu, Y., Geng, P., Shao, Q., Wei, Y. (2017).
Isolation of four phenolic compounds from Mangifera indica, L flowers by using normal phase combined with elution extrusion two-step high speed countercurrent chromatography. J Chromatogr B. 1046, 211-217.

[12] Sheldrick G, M. (2015). Crystal structure refinement with SHELXL. Acta Crystallography C. 71, 3-8. 